ORIGINAL ARTICLE

\title{
Outcome of non-invasive positive pressure ventilation in paediatric neuromuscular disease
}

\author{
S Katz, H Selvadurai, K Keilty, M Mitchell, I MacLusky
}

Arch Dis Child 2004;89:121-124. doi: 10.1136/adc.2002.019562

See end of article for authors' affiliations

.....................

Correspondence to: Dr I MacLusky, Division of Respiratory Medicine, Gerrard Wing, Room 4534, The Hospital for Sick Children, 555 University Avenue, Toronto, Ontario, M5G 1X8, Canada; ian.maclusky@sickkids.ca

Accepted 14 March 2003
Background: Non-invasive positive pressure ventilation (NPPV) has a beneficial effect on nocturnal hypoventilation and hospitalisation rates in adults with static or slowly progressive neuromuscular disease and respiratory failure. Its role in children affected with similar disease processes, however, remains unclear.

Aims: To investigate the impact of NPPV on hospitalisations and sleep related respiratory parameters in children with neuromuscular disease.

Methods: Fiffeen children (mean age 1 1.7, range 3.4-17.8 years) diagnosed with neuromuscular disease who had been started on nocturnal NPPV and had at least one year of follow up since the initiation of such therapy were studied. Patients served as their own controls and comparison was made of the years preceding and following the initiation of NPPV.

Results: Children spent $85 \%$ fewer days in hospital (mean pre-NPPV 48.0 days, mean post-NPPV 7.0 days) and $68 \%$ less days in intensive care after initiation of NPPV (mean pre-NPPV 12.0 days, mean post-NPPV 3.9 days). Sleep study parameters including number of desaturations, apnoea-hypopnoea index and transcutaneous $\mathrm{pCO}_{2}$ levels improved after initiation of NPPV.

Conclusions: NPPV can decrease hospitalisations for children with neuromuscular disease and improves sleep related respiratory parameters. A prospective study is now needed to further delineate the role of NPPV in this population of children.
C ongenital neuromuscular disorders which are complicated by progressive involvement of the respiratory muscles will ultimately lead to chronic respiratory insufficiency, producing significant morbidity and mortality. This respiratory insufficiency may present initially with recurrent chest infections secondary to retained secretions and atelectasis, necessitating frequent hospital admissions, and often requiring intensive care management. As the neuromuscular disease progresses nocturnal respiratory dysfunction becomes evident, ${ }^{1}$ secondary to a combination of decreased muscle tone and reduced sensitivity of the respiratory centres to carbon dioxide during sleep. ${ }^{2}$ Initially the consequent hypoventilation results in transient hypercapnia during sleep that is associated with frequent arousals, and secondary sleep fragmentation. With progression of the neuromuscular disease, this hypoventilation becomes chronic, resulting in daytime hypercapnia. These effects can to a large degree be ameliorated using non-invasive ventilation during sleep. Studies in adults with neuromuscular disease have documented decreased rates of hospitalisation ${ }^{3}$ and have documented improvement in sleep parameters and oxygenation, ${ }^{2-6}$ through the use of nocturnal non-invasive positive pressure ventilation (NPPV).

NPPV appears to have an established role in adults with static or slowly progressive disease. Its role in children affected with progressive, and ultimately fatal neuromuscular disease, however, remains unclear. Benefit has been shown in such children using NPPV for respiratory support in acute illness, ${ }^{78}$ and improvement has been shown in nocturnal oxygen saturation up to eight months after initiation of nocturnal NPPV. ${ }^{9}$ To date, however, there are no long term studies in children evaluating the impact of nocturnal NPPV on hospitalisation rates and intensive care stay for respiratory complications of neuromuscular disease.

Based on evolving evidence of its benefit in paediatric acute respiratory failure and personal experience, the Division of
Respiratory Medicine has implemented the practice of offering NPPV to all patients with neuromuscular disease complicated by hypoventilation or recurrent atelectasis/ pneumonia. In this study we report on the Hospital for Sick Children's experience using NPPV in a cohort of 15 patients with neuromuscular disease. We hypothesised that in the year after initiation of nocturnal NPPV, patients will have had fewer hospitalisations, days in hospital, and ICU visits, in addition to improved nocturnal oxygen saturation and sleep related apnoea-hypopnoea index.

\section{MATERIALS AND METHODS}

A retrospective review was undertaken of children diagnosed with neuromuscular disease followed at the Hospital for Sick Children, who had been started on nocturnal NPPV.

Inclusion criteria: All such patients referred for sleep study with evidence of progressive respiratory insufficiency confirmed either by sleep related hypoventilation on sleep study or hospitalisation due to respiratory compromise, who had at least one year of follow up since the initiation of such therapy were included. Patients served as their own controls and comparison was made of the years preceding and years following the initiation of NPPV.

Exclusion criteria: Patients with neuromuscular disease not receiving nocturnal ventilation and those with bronchopulmonary dysplasia and congenital heart disease were excluded from the study. Infants with spinal muscular atrophy type I were also excluded from this study, since the rapidity of expected clinical decline precludes the ability to assess therapeutic effect over a two-year period.

Abbreviations: NPPV, non-invasive positive pressure ventilation; SMA, spinal muscular atrophy 
NPPV was initiated based on (a) evidence of sleep related hypercapnia $\left(\mathrm{PaCO}_{2}\right.$ greater than $\left.50 \mathrm{mmHg}\right)$ with desaturations to below $92 \%(\mathrm{n}=10)$ or $(\mathrm{b})$ a history of recurrent hospitalisations for pneumonia and/or atelectasis $(n=5)$. Patients received nocturnal NPPV (either by mask or nasal pillows). NPPV devices used in our laboratory consisted of the Harmony, Synchrony, or ST30 (Respironics, USA) BiPAP units. Initial settings were individualised to each patient, based on age, weight, and severity of scoliosis (range IPAP 9$20 \mathrm{~cm} \mathrm{H}_{2} \mathrm{O}$, EPAP 3-6 $\mathrm{cm} \mathrm{H}_{2} \mathrm{O}$ ). Ventilation settings were then titrated in the sleep laboratory to normalise the respiratory pattern and oxygen saturation, and to produce a decrease of at least $10 \mathrm{mmHg}$ in $\mathrm{PaCO}_{2}$, if the transcutaneous $\mathrm{pCO}_{2}\left(\mathrm{Ptc}_{\mathrm{CO} 2}\right)$ was greater than $50 \mathrm{mmHg}$. Patients were breathing spontaneously, with a back up ventilation rate set at least $10 \%$ below the child's own resting breathing rate.

Initiation of NPPV and its titration was performed in the sleep laboratory on an outpatient basis, except when the patients were already hospitalised, in which case it was done during the course of their admission, prior to discharge. There was therefore no increase in hospitalisations for the purpose of initiating or titrating NPPV.

Demographic data including age, sex, diagnosis, and age at initiation of NPPV were collected. Outcome measures were the number of hospital admissions for respiratory complications before and after initiation of NPPV, total number of days in hospital, number of days in intensive care unit, and need for invasive ventilation. These were obtained by audit of the patients' hospital charts. Information about admissions to other hospitals was obtained via telephone interview with the patients' parents and has been included in this analysis. Two of the families could not be contacted and there was therefore potential for unreported hospitalisations. Since the Hospital for Sick Children is the only tertiary level paediatric hospital for the community, it is unlikely that ICU admissions were missed. The decision to admit a patient to hospital was made by the patients' attending physician (not the study physicians) on clinical grounds.

Polysomnography was performed in the sleep laboratory, according to standards of the American Thoracic Society. ${ }^{10}$ Baseline oxygen saturation and transcutaneous $\mathrm{pCO}_{2}$ were also recorded. Sleep study parameters were analysed for the apnoea-hypopnoea index, number, and degree of desaturations. An apnoea was defined as a cessation of airflow with duration of greater than 10 seconds. A hypopnoea was defined as a reduction in airflow or chest wall motion resulting in a decrease in arterial oxygen saturation of at least $4 \%{ }^{10}$ The apnoea-hypopnoea index was calculated as the number of apnoeas plus the number of hypopnoeas divided by the total sleep time in hours.

Outcomes were compared over a one year period prior to onset and post-initiation of NPPV. Paired $t$ tests, pre- and post-initiations of NPPV, were used to analyse the data.

Questionnaires regarding daytime functioning were prospectively collected when patients presented to the sleep laboratory for assessment before and after initiation of NPPV. The questions assessed school performance, appetite, morning headache, and bedwetting.

\section{RESULTS}

Fifteen children with neuromuscular disease of various aetiologies were studied. They included four children with spinal muscular atrophy (SMA) type II, four with muscular dystrophy, of which one has Duchenne's, two children with myotonic dystrophy, and two with nemaline rod myopathy. The other three children had other forms of myopathy, some of whom had not been given a clearly defined diagnosis. The mean age of the patients was 11.7 years (range 3.4-17.8 years). Table 1 outlines the demographics.
We found a significant decrease in the total number of days in hospital and days spent in the intensive care unit after the initiation of NPPV (table 2). Children spent $85 \%$ fewer days in hospital (mean pre-NPPV 48.0 days, mean post-NPPV 7.0 days, $\mathrm{p}<0.001)$ and $68 \%$ less days in intensive care after initiation of NPPV (mean pre-NPPV 12.0 days, mean post-NPPV 3.9 days, $\mathrm{p}<0.015)$ after initiation of NPPV (table 2).

Sleep study data were available for nine of the 15 children after the initiation of NPPV (table 2). There was a $92 \%$ decrease in the number of desaturation events (mean preNPPV 9.7, mean post-NPPV 1.1, p <0.039) in these patients. The documented desaturations were also less severe and the apnoea-hypopnoea index improved. Transcutaneous $\mathrm{pCO}_{2}$ levels also normalised after initiation of NPPV (table 2).

Finally, prospectively administered questionnaires were filled out by seven school aged children at the time of initiation of NPPV. Two children reported improved school performance and fewer morning headaches; five reported no change over the study period. One child had improved urinary continence after starting nocturnal NPPV. None of the children reported deterioration in any of the functions asked on the questionnaire.

\section{DISCUSSION}

This study describes the impact of NPPV on hospitalisation rates and duration in a cohort of children with neuromuscular disease. It is the largest reported study in this paediatric population. Data were collected for one year prior to and after initiation of NPPV. The principle finding was a significant decrease in the days spent in hospital. This supported our previous clinical impression and confirms similar reported results in an adult study. ${ }^{3}$ The magnitude of the decrease is significant, considering that the majority of children in this cohort suffer from progressive disease processes. Thus, an increase in days spent in hospital would be expected as the natural history of these diseases unfolds. The prospective questionnaire administered revealed that some patients report an improvement in daytime function after initiation of NPPV.

The heterogeneity of the study subjects does represent a limitation of this study, since different diseases have different rates of progression. The majority of patients included in this cohort, however, suffered from either myotonic dystrophy or spinal muscular atrophy type II, in which progressive decline in respiratory function over time is expected. Those with SMA type II have a reported $31.5 \%$ mortality rate within the first 25 years of life. ${ }^{11}$ Similarly, patients with congenital myotonic dystrophy have only a $50 \%$ survival into the mid$30 \mathrm{~s}^{12}$ The number of patients with non-progressive myopathy, such as nemeline rod myopathy, was small. It can therefore be expected that as a group, a decline in respiratory function over time would occur and the need for hospitalisation would increase.

Since the accepted practice was to offer NPPV to this population, it was not possible to have a group of true matched controls. In an attempt to address this methodological problem, the authors have used each patient as their own control, comparing the year preceding and following initiation of NPPV.

The study may be biased by changes in hospital and intensive care admission policy over this time period. Initially, all children requiring any form of ventilation assistance were admitted to the intensive care unit for management. Over the last few years, however, there has been a shift in hospital policy, such that children who receive NPPV at home are now being managed on the general paediatric wards. This would have led to a reduction in ICU days in patients more recently enrolled in the programme. It 
Table 1 Baseline descriptive data

\begin{tabular}{|c|c|c|c|c|c|}
\hline Patient & Gender & Diagnosis & Age & $\begin{array}{l}\text { Age at initiation } \\
\text { of NPPV }(y)\end{array}$ & $\begin{array}{l}\text { Duration of } \\
\text { follow up (y }\end{array}$ \\
\hline 1 & $\mathrm{~F}$ & Spinal muscular atrophy type II & 3.83 & 2.6 & 1 \\
\hline 2 & $\mathrm{~F}$ & Congenital muscular dystrophy & 11.25 & 7 & 4.2 \\
\hline 3 & $\mathrm{~F}$ & Congenital fibre disproportionate type myopathy & 6.21 & 1.1 & 4.9 \\
\hline 4 & $\mathrm{~F}$ & Myotonic dystrophy & 15.21 & 13.5 & 1.5 \\
\hline 5 & $\mathrm{~F}$ & Fascio-scapular humeral myopathy & 13.4 & 7.9 & 5 \\
\hline 6 & M & Congenital muscular dystrophy & 13.2 & 10.9 & 1.2 \\
\hline 7 & $\mathrm{~F}$ & Myotonic dystrophy & 17.78 & 13.2 & 3 \\
\hline 8 & $M$ & Spinal muscular atrophy type II & 16.46 & 7 & 8.6 \\
\hline 9 & M & Duchenne muscular dystrophy & 16.79 & 14.3 & 2.4 \\
\hline 10 & M & Spinal muscular atrophy type II & 10.22 & 8 & 2 \\
\hline 11 & M & Nemaline rod myopathy & 14.23 & 5 & 8.5 \\
\hline 12 & $\mathrm{~F}$ & Muscular dystrophy & 9.55 & 6.5 & 3 \\
\hline 13 & $\mathrm{~F}$ & Nemaline rod myopathy & 3.4 & 2 & 1.3 \\
\hline 14 & $\mathrm{~F}$ & Spinal muscular atrophy type II & 6.09 & 0.5 & 4.4 \\
\hline 15 & M & Charcot Marie tooth myopathy & 17.21 & 10 & 7 \\
\hline
\end{tabular}

would not, however, have had any impact on overall hospital days.

An area of debate is the variability in indications for initiating NPPV in children with chronic respiratory insufficiency. A European Consensus Conference has suggested instituting NPPV in patients with neuromuscular disorders who experience morning headache, daytime hypersomnolence, nocturnal sleep difficulties, or cognitive dysfunction. ${ }^{13}$ In the absence of symptoms, a $\mathrm{PaCO}_{2}$ higher than $45 \mathrm{mmHg}$ or $\mathrm{PaO}_{2}$ less than $60 \mathrm{~mm} \mathrm{Hg}$ on morning arterial blood gas sample, or pronounced nocturnal desaturations, are also considered indications to initiate therapy.$^{13}$ These parameters, however, are less well defined in children, who may not be able to vocalise their symptoms and who are not routinely subjected to arterial blood gases. A study by Khan and colleagues, ${ }^{9}$ reporting experience in ambulatory children with congenital neuromuscular disorders used chest infections, morning headache, daytime lethargy, poor weight gain, and hypoxaemia as their indications for treatment. They concluded that treatment with nocturnal NPPV resulted in normalisation of nocturnal oxygen saturation and reduced the number of wake epochs during sleep. In adults, noninvasive intermittent positive pressure ventilation has been shown to be effective in normalising sleep disordered breathing, ${ }^{2}$ possibly by enhancing respiratory control.

As this was a retrospective study, it is difficult to comment on the impact of NPPV on quality of life or compliance with therapy. After initiation of NPPV, it was, however, up to the children and their parents as to whether to persevere with this therapy. The fact that the parents and children reported ongoing use of NPPV would suggest that they themselves perceive a therapeutic benefit.
While the results of this study are encouraging and NPPV seems to provide a solution to some of these children's problems, it is also important to realise that it is not an innocuous treatment. NPPV treatment can be complicated by aspiration pneumonia, mechanical failure of the ventilatory unit, and discomfort related to the fit of the mask. ${ }^{5}{ }^{14}$ Despite these problems, however, a long term study in adults using NPPV for musculoskeletal disorders found that for most patients the benefits far outweighed the risks. $^{5}$

In conclusion, NPPV can increase the quality of life for children with neuromuscular disease, both by decreasing hospitalisations and improving daytime functioning. Such therapy has been controversial in this population of patients, with claims that it only prolonged inevitable death from these diseases, with medical costs associated with deteriorating respiratory function only being deferred. While NPPV is not a cure for these diseases, empirically it appears to have a substantial positive impact on patients with neuromuscular disease. These disorders do lead to progressive loss of muscle function, and eventual death from respiratory failure. The aim of this programme was to increase both quality and duration of life, normalising, as much as possible, the child's day to day existence and when appropriate, having death occur in the community setting. Based on the results of this retrospective study, a randomised control trial would be difficult to ethically justify. A prospective study, however, needs to be undertaken to further define the precise role of NPPV in children with neuromuscular diseases, specifically with respect to indications and timing for initiation of this therapy, and its impact on quality of life for both children and caregivers.

Table 2 Comparative analysis; pre- $v$ post-onset of NPPV $\dagger$

\begin{tabular}{|c|c|c|c|}
\hline Clinical outcome & $\begin{array}{l}\text { One year pre-NPPV } \\
\text { Mean (SEM) }\end{array}$ & $\begin{array}{l}\text { Annual decrease } \\
\text { post-NPPV } \\
\text { Mean (SEM) }\end{array}$ & $95 \% \mathrm{Cl}$ \\
\hline No. of admissions to hospital per year & $4.1(0.7)$ & $3.1(0.8)^{*}$ & 1.3 to 4.8 \\
\hline No. of days in hospital per year & $48.0(10.5)$ & $41.0(8.6)^{* *}$ & 22.6 to 59.4 \\
\hline No. of admissions to ICU & $1.1(0.2)$ & $0.7(0.2)$ & 0.2 to 1.2 \\
\hline No. of days in ICU per year & $12.0(3.5)$ & $8.1(2.9)^{*}$ & 1.8 to 14.4 \\
\hline No. of nocturnal desaturations & $9.7(3.4)$ & $8.9(3.6)$ & 0.6 to 17.2 \\
\hline Apnoea-hypopnoea index & $7.3(3.5)$ & $4.5(1.2)$ & 1.7 to 7.4 \\
\hline Minimum nocturnal oxygen saturation & $66.2(6.9)$ & $-22.0(7.1)^{*}$ & -38.4 to -5.6 \\
\hline Depth of nocturnal desaturations & $27.9(6.5)$ & $21.4(6.9)^{*}$ & 5.5 to 37.4 \\
\hline Baseline $\mathrm{CO}_{2}$ & $69.2(4.1)$ & $16.3(4.0)^{*}$ & 7.1 to 25.6 \\
\hline
\end{tabular}

†Data compare the year prior to and post-initiation of NPPV.

${ }^{*} \mathrm{p}<0.05,{ }^{* *} \mathrm{p}<0.01$. 


\section{Authors' affiliations}

S Katz, H Selvadurai, K Keilty, M Mitchell, I MacLusky, The Hospital for Sick Children, Toronto, Canada

\section{REFERENCES}

1 Bach JR, O'Brien R, Krotenberg, et al. Management of end stage respiratory failure in Duchenne muscular dystrophy. Muscle Nerve 1987;10:177-82.

2 Barbe F, Quera-Salva MA, de Lattre J, et al. Long-term effects of nasal intermittent positive pressure ventilation on pulmonary function and sleep architecture in patients with neuromuscular diseases. Chest 1996; 110:1 179-83.

3 Tzeng AC, Bach JR. Prevention of pulmonary morbidity for patients with neuromuscular disease. Chest 2000;118:1390-6.

4 Hukins CA, Hillman DR. Daytime predictors of sleep hypoventilation in Duchenne muscular dystrophy. AJRCCM 2000;161:166-70.

5 Baydur A, Layne E, Aral H, et al. Long term non-invasive ventilation in the community for patients with musculoskeletal disorders: 46 year experience and review. Thorax 2000;55:4-11.

6 Guilleminault C, Philip P, Robinson A. Sleep and neuromuscular disease: bilevel positive airway pressure by nasal mask as a treatment for sleep-disordered breathing in patients with neuromuscular disease. J Neurol Neurosurg Psychiatry 1998;65:225-32.

7 Padman R, Lawless ST, Kettrick RG. Noninvasive positive pressure ventilation via bilevel positive airway pressure support in pediatric practice. Crit Care Med 1998;26:169-73.

8 Niranjan V, Bach JR. Noninvasive management of pediatric neuromuscular ventilatory failure. Crit Care Med 1998;26:2061-5.

9 Khan Y, Heckmatt JZ, Dubowitz V. Sleep studies and supportive ventilatory treatment in patients with congenital muscle disorders. Arch Dis Child 1996;74:195-200.

10 Anon. Standards and indications for cardiopulmonary sleep studies in children. American Thoracic Society. AJRCCM 1996;153:866-78.

11 Zerres K, Rudnik-Schoneborn S, Forrest E, et al. A collaborative study on the natural history of childhood and juvenile onset proximal spinal muscular atrophy (type II and III SMA). J Neurol Sci 1997;146:67-72.

12 Reardon W, Newcombe R, Fenton I, et al. The natural history of congenital myotonic dystrophy: mortality and long term clinical aspects. Arch Dis Child 1993;68:177-81

13 Robert D, Willig TN, Leger P. Long-term nasal ventilation in neuromuscular disorders: report of a consensus conference [published correction appears in Eur Respir J 1993;6:1233] Eur Respir J 1993;6:599-606.

14 Lechtzin N, Weiner CM, Clawson L. A fatal complication of noninvasive ventilation. N Engl J Med 2001;344:533.

\section{ARCHIVIST}

\section{Tobacco, alcohol, cannabis, and adolescent mental health}

Y oung adolescents who smoke regularly are more likely than non-smokers to have mental health problems. This is the main finding of a study done in 1999 through the Office for National Statistics (A Boys and colleagues. British Journal of Psychiatry 2003;182:509-17).

The study included 2624 children aged 13-15 years who lived in 475 different postal sectors in England, Scotland, and Wales. Interview data, including psychiatric assessment and information about use of tobacco, alcohol, and cannabis, were collected from 2458 children. A psychiatric diagnosis was made for 307 children (12\%) and categorised as depressive disorder (66 children), other emotional disorder (104), or other (non-emotional) disorder (137). The 1964 children (80\%) who had never tried cannabis and who were not regular smokers or drinkers formed a comparison group. The 495 who smoked regularly, drank (at least once a week), or had ever used cannabis (or came into any combination category) were the study group.

The three substance use categories were interrelated so that use of any one substance increased the likelihood of use of either or both of the others. For example, regular drinkers were eight times more likely than non-drinkers to be regular smokers. Smoking alone increased the risk of a psychiatric diagnosis by a factor of five and having ever used cannabis doubled the risk. Weekly use of alcohol did not increase the risk. Regular smoking combined with ever use of cannabis increased the risk sevenfold. The 53 children who came into all three substance use categories had a 14 -fold increase in risk of any psychiatric diagnosis and a 27-fold increase in risk of depressive disorder. Smoking was most strongly associated with psychiatric disorder and regular (as opposed to ever) use of cannabis was less so. Alcohol use was not associated with psychiatric diagnosis. The three categories of substance use, however, seem ill-assorted as regards dosage; "regular drinking" included any child who admitted drinking alcohol once a week or more and the cannabis use category included those who had tried it once. Regular drinking was commoner in children from more affluent families but regular smoking was commoner in poorer families.

There is a connection between smoking and psychiatric problems in early adolescence but the nature of the connection seems unclear. 